\title{
LA PALESTINE, LES ARABES ET LE MONDE ARABE : ENTRE RIVALITÉS ET REPRÉSENTATION
}

Entretien avec Leïla Shahid, propos recueillis par Béatrice Giblin, Pierre Blanc

\author{
La Découverte | « Hérodote »
}

2016/1 N 160-161 | pages 273 à 286

ISSN 0338-487X

ISBN 9782707189417

Article disponible en ligne à l'adresse :

https:/www.cairn.info/revue-herodote-2016-1-page-273.htm

Distribution électronique Cairn.info pour La Découverte.

(C) La Découverte. Tous droits réservés pour tous pays.

La reproduction ou représentation de cet article, notamment par photocopie, n'est autorisée que dans les limites des conditions générales d'utilisation du site ou, le cas échéant, des conditions générales de la licence souscrite par votre établissement. Toute autre reproduction ou représentation, en tout ou partie, sous quelque forme et de quelque manière que ce soit, est interdite sauf accord préalable et écrit de l'éditeur, en dehors des cas prévus par la législation en vigueur en France. Il est précisé que son stockage dans une base de données est également interdit. 


\title{
La Palestine, les Arabes et le monde arabe : entre rivalités et représentation
}

\author{
Entretien avec Leïla Shahid, \\ ex-ambassadrice de l'OLP \\ (Organisation de libération de la Palestine) \\ auprès de la France puis de l'Union européenne
}

Hérodote - Que représente aujourd' hui le monde arabe? Existe-t-il, et si oui, qu'est-ce qui en fait l' unité et le caractérise?

Leïla Shahid - Quand on parle du monde arabe qui n'est pas monolithique, il faut savoir si l'on parle des États qui le composent ou des peuples. Avec cette crise profonde, à la fois sociale et civilisationnelle, le monde arabe existe plus que jamais, au moins au niveau des sociétés civiles qui expriment de fait leur bonne santé. Désormais, depuis le début des soulèvements en 2011, elles vivent un véritable séisme et c'est une nouvelle phase de leur histoire, voire une mutation, qui s'amorce. Par ailleurs, on assiste à la décomposition de certains pays comme l'Irak et la Libye, notamment du fait des interventions américaine et européenne.

En disant cela, je tiens à préciser que je ne suis pas une chercheuse mais une militante diplomate qui a sillonné le monde arabe en long et en large. J'ajouterai aussi que je suis palestinienne, née au Liban, et que j'ai beaucoup voyagé dans les pays du Maghreb et du Machrek. C'est donc à partir de mon expérience et de mon vécu que je m'exprime ici sur la réalité du monde arabe.

Pour revenir à cette crise très profonde et historique que traverse le monde arabe, je dirai qu'elle est en fait la première depuis les indépendances. La décolonisation, au lendemain de la Seconde Guerre mondiale et jusqu'au début des années 1960, avait constitué une étape importante sur le chemin de l'émancipation et de la souveraineté. Mais le temps a montré qu'il s'agissait en fait pour la majorité des vingt-deux États de la Ligue arabe d'une pseudo-souveraineté, tant 
sur le plan économique et constitutionnel que sur celui des libertés publiques et des droits civils. Au moment des indépendances, peu de structures démocratiques furent mises en place et la séparation des pouvoirs et la liberté de presse étaient limitées. Aussi l'espoir des indépendances s'est-il progressivement mué en une grande déception pour les citoyens, car ils se sont trouvés dépossédés par leurs gouvernements successifs de leur droit à participer à la vie démocratique de la société civile et à la construction de leur État. Les logiques de la guerre froide avec son lot d'ingérences n'ont pas facilité non plus cette transition. Ce contexte n'a pas aidé la convergence des vingt-deux États de la Ligue arabe qui ont pourtant beaucoup plus en commun que les vingt-huit de l'Union européenne, comme la langue, l'histoire, la culture, et cela même entre les Arabes et les Berbères du Maghreb, ou entre les chrétiens et les musulmans du Machrek. Mais, si les États de l'UE ont la volonté de construire un espace commun, tel n'est pas le cas entre les États du monde arabe. La preuve, c'est que les frontières sont assez fermées, et qu'il n'y a ni marché commun, ni règle douanière commune. Pourtant, dans les sociétés arabes, il y a un sentiment très profond d'appartenir à un même ensemble, à une identité et à une histoire communes, à un ensemble régional de 300 millions d'habitants qui contrôle les ressources pétrolières les plus importantes au monde et qui doit jouer un rôle dans la mondialisation.

La crise en cours, ce séisme même, est donc une nouvelle phase dans l'évolution du monde arabe. Les «printemps arabes», qui se sont influencés les uns les autres, soulignant une arabité commune, ont mis en exergue la vitalité de la société civile même si la victoire n'est pas encore là. On a assisté à l'explosion de révoltes citoyennes organisées ni par des partis ni par des syndicats mais par des mouvements sociaux. Ces mouvements étaient d'une modernité spectaculaire. Ils étaient laïques, profondément non religieux, et ont touché l'ensemble du monde arabe. Mais n'étant pas organisées par les partis politiques, ces révoltes ont fait peur et les institutions militaires ou religieuses en ont repris le contrôle en passant des alliances factices.

Les échecs du monde arabe depuis les indépendances conduisent actuellement au retour de la religion et à son instrumentalisation. En Palestine, on observe le même phénomène, ce qui constitue un réel problème car pour arriver à la création de deux États, il faut reconnaître l'altérité. Or, avec la religion comme cadre exclusif du politique, il n'y a pas d'altérité car sa reconnaissance passe nécessairement par la laïcité. Cette affirmation actuelle du religieux ne concerne d'ailleurs pas uniquement l'islam: elle concerne aussi certains juifs religieux qui exercent une influence croissante sur le pouvoir en Israël. Dans le monde arabe, le discours religieux est une réponse à l'échec de savoir penser l'altérité. Or, dans l'Empire ottoman, en Palestine et surtout dans le sandjak de Jérusalem on a vécu l'altérité entre juifs, chrétiens et musulmans pendant plusieurs siècles. 
Par-delà la résistance des institutions au changement, il faut ajouter que l'intervention américaine et européenne en Irak et en Libye a ouvert la boîte de Pandore et encouragé dans le premier cas un affrontement entre chiites et sunnites et, dans le second, des guerres tribales.

Considérant cet échec des printemps arabes, je pense qu'il n'est en rien définitif à condition qu'on prenne en compte le temps long. La crise de légitimité des dirigeants n'est tout simplement pas terminée, sauf en Tunisie, seul État arabe où la démocratie fonctionne grâce à une société civile plus développée et vigilante, et où une coalition entre laïcs et islamistes a pu être réalisée, ce qu'en Palestine on a été incapables de faire. Bien sûr, la situation de la Tunisie reste fragile et c'est d'ailleurs pour cela que je me réjouis que le prix Nobel de la paix lui ait été donné.

Dans le monde arabe, je reste encore convaincue qu'il y a un contraste énorme entre la profonde crise de légitimité des États et l'énorme vitalité des sociétés civiles qui représentent un potentiel fantastique, en particulier les jeunes et les femmes, qui sont créatifs et très capables d'apprendre de leurs propres erreurs. Le potentiel de soulèvement, intifada, n'est pas épuisé dans le monde arabe avec les spécificités de chacune des sociétés et de chacun des pays.

\section{Vous revenez sur ce terme d'intifada qu' on associe à la Palestine.}

L. S. - Et pour cause! Ce mot qui vient du verbe intafada signifiant se secouer, au sens de décider de se bouger, a été utilisé en premier dans le cas palestinien. Si les premières opérations militaires à l'occupation israélienne ont démarré en 1956 à Gaza, où les réfugiés palestiniens chassés par les Israéliens étaient très nombreux, la première intifada eut lieu en 1969, c'est-à-dire deux ans après la terrible défaite de 1967, dans les camps de réfugiés du Liban.

Je rappelle le contexte: 1967, c'est la débâcle, la défaite humiliante, l'équivalent de la défaite de 1940 pour la France. J'avais 18 ans, j'habitais Beyrouth, c'était le matin de la première épreuve du baccalauréat et l'annonce de la guerre donna lieu à la suspension des épreuves. Nous étions absolument convaincus que nous allions la gagner en deux temps, trois mouvements. Qui plus est, c'était pour nous la revanche de la défaite des armées arabes en 1948 face à l'armée israélienne. Et six jours plus tard, on faisait le constat d'un immense désastre, la «nakba». Dans le monde arabe, l'issue de cette guerre fut ressentie comme une perte de dignité. Non seulement Israël avait conquis de nouveaux territoires arabes, mais en plus on avait montré qu'on ne savait pas utiliser l'armement moderne à notre disposition, notamment l'aviation égyptienne. À ce moment-là, les quelques Palestiniens qui lancèrent la lutte contre les Israéliens furent vus comme des héros car ils osèrent agir là où les armées arabes avaient été défaites et humiliées. C'est la bataille de Karameh en Jordanie qui est en quelque sorte restauratrice de la 
dignité perdue (la coïncidence veut que Karameh signifie dignité). Cette bataille faisait suite à un attentat du Fatah en mars 1968 qui poussa l'armée israélienne à attaquer la ville de Karameh. Si cette bataille est devenue légendaire dans l'histoire palestinienne, mais aussi dans le monde arabe, c'est bien parce que les «fedayins » surent alors affronter l'armée israélienne, redonnant ainsi aux Arabes leur fierté et leur dignité. Grâce à cette bataille les fedayins et le Fatah avaient acquis une aura dans la population arabe qui allait se traduire par un accroissement du nombre des partisans venus de plusieurs pays arabes, assurant au Fatah une dimension internationaliste.

Au Liban, il y avait onze camps de réfugiés. Ils étaient contrôlés par le deuxième bureau de l'armée libanaise (les services de sécurité) car les réfugiés palestiniens étaient jugés comme des étrangers dangereux et subversifs. Ces camps palestiniens étaient inaccessibles aux citoyens libanais mais l'OLP entreprit un travail politique clandestin avec la gauche libanaise. Et Arafat, à la tête de l'OLP depuis 1969, sut profiter du large soutien dans le monde arabe pour se débarrasser du contrôle du deuxième bureau libanais. La population des camps sortit alors dans les rues sans arme, sans violence et le rapport de force, plus symbolique que militaire, était tel que la sécurité libanaise quitta les camps. L'organisation politique palestinienne se mit alors en place avec l'établissement des comités populaires composés de représentants des camps. Le premier nom donné à ce mouvement fut «intifada» et on peut le considérer comme le réveil citoyen palestinien. C'était la première victoire politique de l'OLP.

En 1987, quand démarre la révolte palestinienne dans les territoires occupés, le terme intifada est repris, c'est comme un passage de relais. On est sous occupation israélienne depuis vingt ans. Au Liban, les Palestiniens ont affronté le siège de Beyrouth, notamment avec les massacres de Sabra et Chatila en 1982, puis l'occupation par l'armée syrienne pendant trois ans. S'il faut donc attendre 1987 pour voir le réveil dans les territoires occupés, c'est en partie dû au fait que, à la différence des mouvements de libération nationale vietnamien ou algérien, toute la direction politique du mouvement de libération palestinien était alors à l'extérieur, c'est-à-dire dans les camps de réfugiés, libanais, jordaniens et syriens. Bien que basée essentiellement sur des mobilisations civiles, cette intifada qui dure jusqu'en 1993 a été puissante car elle a contribué à la reconnaissance de l'OLP et au déclenchement du processus d'Oslo. En comparaison, la dernière intifada, née d'une réponse à la provocation d'Ariel Sharon le 28 février 2000, a été une catastrophe car elle n'a pas été gérée par la société civile mais militarisée par certaines institutions, s'offrant ainsi à l'immense répression de l'armée israélienne que voulait Sharon. 
En quoi la question de Palestine a-t-elle été un facteur d'identification pour le monde arabe?

L. S. - La Palestine occupe une place très particulière dans la mémoire des Arabes, dans leur psyché car c'est la terra santa, la terre sainte comme le rappelait toujours Arafat. C'est aussi le symbole de la lutte anticoloniale dans le monde arabe pour la justice et le droit. Sur le plan de la mémoire historique, la Palestine symbolise aussi la reconnaissance de l'altérité. Au début du XX ${ }^{\mathrm{e}}$ siècle, la population palestinienne était composée majoritairement de musulmans mais aussi de chrétiens et de juifs du Yichouv, essentiellement orthodoxes et donc hostiles au mouvement sioniste. C'est une expérience historique d'une immense richesse. Sur le plan économique, la Palestine était, avant l'occupation britannique et l'arrivée des colons sionistes, potentiellement riche: Haïfa était un port bien plus important que Beyrouth avec des relations commerciales et diplomatiques avec l'Europe. Il y avait déjà un consul de la Palestine à Bordeaux marié à une Française et qui avait fait ses études à la Sorbonne. Sur le plan culturel, c'est en Palestine qu'ont été traduits pour la première fois dans le monde arabe des ouvrages de la philosophie des Lumières. Faut-il rappeler aussi que la Palestine fut un lieu important de la renaissance, nahda, arabe? De même, il faut dire que Jérusalem était déjà une ville cosmopolite du fait des Lieux saints et donc de la présence de nombreuses missions étrangères et de l'accueil de pèlerins de différentes religions. Et parmi ces pèlerins il y avait aussi des archéologues, des linguistes spécialisés dans des langues telles que l'araméen, mais aussi des voyageurs célèbres tels que Chateaubriand et Lamartine qui ont mis en lumière la vie en Palestine. Non seulement la vie urbaine était dynamique à Jérusalem mais elle était très active aussi à Haïfa, Naplouse, Jaffa et Hébron. Il faut rappeler cela car c'est une situation historique méconnue que même les nouveaux historiens israéliens ont sous-estimée. D'ailleurs, on voit toujours les Palestiniens comme étant à cette époque des paysans pauvres ou des Bédouins nomades. C'est bien la fiction inventée par les Européens. Or c'est la colonisation britannique qui a contribué à vider la Palestine de cette vie urbaine et intellectuelle ${ }^{1}$.

Au départ des Britanniques, c'est la Jordanie qui hérite de la Cisjordanie et de Jérusalem-Est et l'Égypte de Gaza, et il y a une totale disparition de 1947 à 1968 de la Palestine, jusqu'au réveil de la lutte palestinienne dans les bases des fedayins en Jordanie, qui a permis de montrer au monde que les Palestiniens et la Palestine existaient. Personne ne peut dire aujourd'hui qu'ils n'existent pas.

1. Voir Elias Sanbar, Figures du Palestinien, Gallimard, Paris, 2004, et Les Palestiniens, la photographie d' une terre et de son peuple de 1839 à nos jours, Hazan, Paris, 2004. Voir aussi Henry Laurens, La Question de Palestine (cinq volumes), Fayard, Paris. 
La Palestine est donc dans les sociétés arabes, et au-delà, emblématique d'un combat juste pour le droit des peuples à disposer d'eux-mêmes, pour le respect du droit international et pour l'indispensable reconnaissance de l'altérité. Á la dimension du mouvement de libération nationale s'ajoutaient en effet la lutte démocratique, l'égalité homme/femme, une Constitution laïque, la liberté de la presse. J'ajouterais que, dans le monde arabe, la mémoire de la lutte coloniale en Palestine est très vive et entretenue et je peux citer les révoltes anticolonialistes de 1936, 1956, 1969, 1987, 2001.

Tout ceci n'épuise cependant pas les ressorts de la question de Palestine chez les Arabes. Elle constitue aussi une blessure profonde dans la psyché arabe qui les renvoie aux siècles de colonisation qu'ils ont subis et d'une injustice jamais acceptée. Elle demeure une plaie ouverte et, où qu'ils soient dans le monde arabe mais aussi en France, les Arabes se reconnaissent à cette blessure ouverte profonde sur le plan émotionnel et producteur de frustration et de violence. Ajoutons enfin qu'en l'absence de structure panarabe la Palestine est la projection de ce qui les unit. Pour toutes ces raisons, elle revêt donc un caractère central pour les peuples arabes qui sont unis autour de la cause palestinienne.

\section{Et quel a été le rôle des États arabes?}

L. S. - Il faut bien distinguer ce que la question de Palestine représente chez les citoyens arabes et ce qu'en ont fait les États qui les représentent. Force est d'admettre que, dans l'échec de la reconnaissance du droit des Palestiniens à leur terre et à la création de leur État, la responsabilité de ces États arabes est grande. Les relations entre les États arabes et la Palestine sont en fait très complexes et ce avant même la création de l'État d'Israël. La promesse de Balfour en 1917 qui prévoit la création d'un foyer national juif en Palestine est le début de la nakba («désastre», «catastrophe» que fut l'exode palestinien). Certes, il stipule qu'il ne faut causer aucun préjudice à la population «non juive», mais cette appellation est des plus surprenantes car le pays est peuplé de Palestiniens! En fait, les Palestiniens réalisent très tôt que les Britanniques et les dirigeants sionistes des pays européens utilisent l'antisémitisme et le contexte de l'effondrement de l'Empire ottoman pour créer un État national juif en Palestine, en prenant appui sur le récit biblique. Au préalable, les sionistes ont pourtant pensé à d'autres pays comme l'Ouganda et l'Argentine. Réalisant progressivement ces desseins britanniques, un mouvement de résistance palestinienne politique, diplomatique et souvent non violent se développe entre 1920 et 1936, notamment du fait des ventes de terres à des Juifs par des propriétaires arabes absentéistes. Mais l'arrivée de colons juifs de plus en plus nombreux et soutenus par le mouvement sioniste suscite l'hostilité des paysans palestiniens qui débouche en 1936 sur la grande révolte. Elle est le fait de paysans armés dirigés par Ezzedine al-Qassam, un 
militant anticolonialiste syrien qui va engager l'insurrection à Haïfa. La répression britannique est très féroce et des lois d'urgence (emergency laws) sont alors mises en place (elles seront reprises plus tard par les Israéliens). Cette révolte est finalement écrasée en 1939, l'élite résistante nationale palestinienne étant alors décapitée, notamment par l'exil de ses dirigeants du Haut comité arabe aux Seychelles, à l'époque sous mandat britannique.

Pendant la Seconde Guerre mondiale, les Palestiniens cherchent à poursuivre la lutte contre les Britanniques et le mouvement sioniste mais les dirigeants arabes des pays déjà indépendants (l'Irak et l'Égypte notamment) ne les arment pas, ce qui explique pourquoi le mufti de Jérusalem, Mohammed Amin al-Husseini, demande de l'aide à Hitler, au nom du principe que «les ennemis de mes ennemis sont mes amis». Il pensait sans doute que des Palestiniens seraient annexés à la brigade bosniaque musulmane mais cette opération visant à obtenir des armes finalement fut sans résultat. Les Palestiniens restaient donc sans force réelle face à la Haganah, organisation clandestine paramilitaire sioniste créée en 1920, et aux milices d'extrême droite, Irgoun et Stern.

Après la Seconde Guerre mondiale, le contexte est marqué par la création d'Israël et l'indépendance de la plupart des États arabes. C'est donc surtout à partir de ce moment-là que le comportement des États arabes sur la question de Palestine peut être analysé. Pour l'essentiel, les États arabes en pointe dans le conflit ont cherché à contrôler le nationalisme palestinien tout en se servant de son écho positif dans les sociétés arabes pour asseoir leur légitimité auprès d'elles. La cause de la Palestine étant devenue le cœur battant du monde arabe, tout dirigeant arabe qui se présente comme son défenseur acquiert une grande légitimité dans la région. Depuis 1945 jusqu'à aujourd'hui, la cause palestinienne est ainsi instrumentalisée par les dirigeants arabes pour acquérir légitimité, crédibilité et respectabilité. Ce fut le cas avec Nasser, puis avec Hussein de Jordanie et Hafez el-Assad. Les Palestiniens ont bien conscience que les dirigeants arabes les utilisent pour servir leur propre stratégie. Cependant, si les Palestiniens ont cherché l'autonomie dans leur lutte de libération, ils n'ont pas pu s'affranchir des pays arabes pour se faire entendre dans les arènes diplomatiques internationales, puisque eux seuls y étaient représentés.

La reprise de quelques éléments historiques de la question de Palestine permet ainsi de souligner cette relation complexe entre États arabes et Palestiniens, où se conjuguent admiration des Palestiniens et instrumentalisation de leur cause.

Dès la création de la Ligue arabe en 1944, certains pays avaient même suggéré aux Palestiniens de n'envoyer personne pour les représenter car la Ligue était supposée le faire. De même, à la création de l'OLP en 1964 à Jérusalem, Nasser avait placé à sa tête Ahmed Choukairi, un homme à sa main, et installé le siège de l'organisation au Caire, pour être sûr d'en garder le contrôle. Le président égyptien 
voulait être le leader du monde arabe et le fait de garder les Palestiniens sous sa coupe servait donc son projet. Nasser a même été jusqu'à accuser les dirigeants palestiniens du Fatah d'être atlantistes. De leur côté, les Jordaniens qui voulaient aussi être les représentants des Palestiniens ont longtemps ignoré l'OLP. Et, en septembre 1970, c'est l'armée jordanienne sur ordre d'Hussein qui affronta les combattants palestiniens, et firent fuir leurs dirigeants vers le Liban. Il a fallu l'intifada de 1987 pour qu'Amman finisse par reconnaître leur autonomie et l'OLP. De même, Hafez el-Assad ne voulait absolument pas aider à la création d'un État palestinien car le parti Baath se voulait le seul représentant des Palestiniens. Je rappelle qu'en 1976, au tout début de la guerre civile libanaise, l'armée syrienne intervient à la demande des phalangistes libanais contre les camps de réfugiés palestiniens puis, à partir de 1982 et jusqu'en 1985, l'armée syrienne assiège les camps palestiniens pour punir Arafat d'avoir choisi de se réfugier à Tunis et non à Damas pourtant très proche $(120 \mathrm{~km})$ quand il a été contraint de quitter Beyrouth avec l'aide de la France, pour entamer une traversée du désert jusqu'en 1993. Cet éloignement avait le mérite de lui permettre de conserver l'autonomie de la décision politique par rapport à certains États du Proche-Orient qui avaient souhaité le contrôler.

Mais même s'ils n'ont pas été réellement soutenus par les États arabes, c'est le moins qu'on puisse en dire, les Palestiniens ont besoin des États arabes car ils n'ont aucune institution officielle et dépendent d'eux pour se faire entendre, par exemple à l'ONU. La situation est donc d'autant plus complexe. Arafat était convaincu que les Palestiniens devaient prendre en charge eux-mêmes leur combat et ne pas le laisser aux États arabes. Comme je l'ai déjà dit, cette volonté des Palestiniens de prendre en main leur combat déplaisait fortement aux pays arabes. Mais jamais Arafat ne s'est départi de sa conviction qu'il était impératif de donner à l'OLP une certaine légitimité arabe et qui, pour ce faire, devait être reconnue comme le seul, l'unique représentant des Palestiniens. Après avoir réussi à intégrer tous les autres mouvements dont les deux principaux, le Front populaire de libération de la Palestine et le Front démocratique pour la libération de la Palestine, l'OLP est enfin reconnue comme seule organisation représentante des Palestiniens par la Ligue arabe en 1974. Ce sera le point de départ d'une chaîne de reconnaissances : les États musulmans de l'Organisation de la conférence islamique puis les pays non-alignés et enfin l'Assemblée générale des Nations unies qui lui accorde le statut d'entité observatrice. Ceci contraint néanmoins les Palestiniens à passer par le groupe des États arabes pour se faire entendre à l'ONU, et ce jusqu'au 29 novembre 2012, où elle est reconnue comme État observateur non membre de l'ONU par 138 voix pour, 9 contre et 41 abstentions. 
Aujourd' hui qu'en est-il des États arabes vis-à-vis de la question de la Palestine et des contacts entre Palestiniens et les autres Arabes?

L. S. - Il faut effectivement faire de nouveau la différence entre les États, les gouvernements et les populations. Les pays du Golfe disent clairement que leur problème actuellement c'est l'Iran et non pas Isrä̈l. Anciens pays du front contre Israël, l'Irak et la Syrie sont en guerre civile, ce qui les détourne de la question de la Palestine. Il en est de même pour le Liban très divisé et faible. Quant au Yémen, il est sur le chemin d'un nouvel Afghanistan. Le seul lieu où l'on retrouve une unanimité arabe officielle pour soutenir les Palestiniens, c'est l'ONU.

Quant aux citoyens du monde arabe, le combat des Palestiniens est aussi leur propre combat pour l'émancipation et la démocratie. Mais toutes les frontières de la Palestine sont des frontières israéliennes. Or les États arabes, sauf l'Égypte et la Jordanie, n'ont aucune relation avec Israël, ce qui contraint les citoyens arabes à ne pas venir en Palestine. Les rencontres ne pouvant se faire qu'ailleurs, les Palestiniens sont très désireux de participer aux différents forums ou biennales artistiques car c'est le moyen de rencontrer d'autres Arabes. Mais pour sortir de chez eux, il faut un permis militaire délivré par les Israéliens qui leur est très souvent refusé sans raison.

Les intellectuels égyptiens pourraient venir puisqu'il y a un traité de paix, mais ils ne viennent pas, pas plus que les coptes, qui pourraient venir prier au SaintSépulcre à Jérusalem, mais leur ancien patriarche Shenouda III le leur avait interdit car pour cela ils doivent traverser des frontières israéliennes. Il en est de même pour les musulmans d'Égypte et de Jordanie. Or la fréquentation de Jérusalem par des étrangers, surtout chrétiens, serait importante pour marquer la pluralité de Jérusalem.

Cependant, cette séparation de fait a pu être dépassée grâce aux chaînes satellitaires. Ainsi, dans les pays arabes, on a su ce qui se passait dans les territoires palestiniens : les Arabes ont vu les trois guerres qui ont touché Gaza, ce qui n'était pas le cas lors de la guerre des Six Jours, ou lors des révoltes palestiniennes dans les camps de réfugiés du Liban en 1968-1969. Grâce aux médias, télévisions, réseaux sociaux, ces liens demeurent les meilleurs antidotes aux frontières et ils permettent de faire vivre cette empathie (tawâçul) vis-à-vis des Palestiniens. Sans eux, les Palestiniens auraient disparu du champ de vision arabe.

Les combattants palestiniens voulaient dépasser les confessions religieuses pour mener unis le combat contre l'occupation israélienne, les succès électoraux du Hamas et la radicalisation islamique d'une partie des Palestiniens changent la donne pour les Palestiniens chrétiens. Qu' en est-il aujourd' hui de leur avenir?

L. S. - La société palestinienne est profondément pluraliste et très attachée à ce pluralisme. Le premier projet de l'OLP était un État démocratique et laïc pour les 
juifs, les chrétiens et les musulmans. Cette société n'est donc pas confessionnalisée comme peut l'être la société libanaise. Elle est issue de la Terre sainte des trois monothéismes et elle assume son enracinement dans toutes les strates historiques de la Palestine. L'identité palestinienne est donc plurielle, tout comme la direction de l'OLP qui a accueilli de nombreux chrétiens. Arafat faisait référence au fait que Jésus était palestinien. Cependant, aujourd'hui, la Palestine ne fait pas exception à l'ambiance régionale qui est marquée par un islamisme rampant. À l'instar des chrétiens d'Orient dont certains fuient de la région ou sont contraints de le faire, il y a une réelle inquiétude chez les chrétiens de Palestine. Il faut quand même souligner que le succès du Hamas en 2006 a été moins une adhésion aux principes islamistes qu'un vote sanction contre le Fatah accusé de corruption et de népotisme et qui, entre 1996 et 2006, n'a pas tenu les promesses faites à la suite des accords d'Oslo. Huit ans après ce succès électoral du Hamas, la population est aussi critique à son sujet qu'elle l'était envers le Fatah. En dehors du Fatah et du Hamas il n'y a aucune autre offre politique, mis à part celles de Fronts populaires et démocratiques qui demeurent assez marginalisés. Pourtant on sent bien dans la société palestinienne le désir de développer un mouvement nouveau qui sache tirer les expériences de toutes les intifada, celle qui fut un succès, la première de 1987-1993, comme celles qui furent des échecs en 2001 et actuellement ce qu'on appelle malencontreusement l'intifada des couteaux. Est-on parvenu à la maturité politique pour créer un mouvement citoyen pluraliste, qui réunisse chrétiens, musulmans et non-croyants, c'est-à-dire un mouvement citoyen comme en Tunisie? Sans doute que oui mais je reconnais qu'une telle mobilisation est très difficile dans un contexte de fragmentation, pour ne pas dire d'atomisation de l'espace et de la société qui est le résultat de la stratégie israélienne justifiée au nom de la sécurité. Depuis 1993, la division de l'espace palestinien en trois types de territoires $(\mathrm{A}, \mathrm{B}, \mathrm{C})$ plus ou moins contrôlés par les Israéliens a fortement contribué à casser le mouvement palestinien. La circulation des Palestiniens d'une zone à l'autre est devenue difficile à cause des checks points, sans parler du mur en certains endroits. Ceci pousse de fait les Palestiniens au repli sur leur village, leur clan, leur famille au sens large, leur tribu. C'est donc à ce niveau très local que se développe le sentiment de loyauté, et non plus envers les institutions politiques, syndicales et culturelles représentant l'ensemble des Palestiniens. Ainsi Israël pense qu'une fois l'OLP détruite, il ne reste que les représentants de village, les chefs locaux pour négocier. Le risque est de voir le retour des ligues de village où certains chefs de village (mukhtar) acceptaient de collaborer avec les Israéliens Ainsi, on empêche d'une manière pernicieuse la survenue de l'État palestinien en détruisant peu à peu le sentiment national palestinien. 
On sait l' implication de la diaspora juive dans le devenir d' Israël. Qu' en est-il de la diaspora palestinienne dans le devenir d' un hypothétique État palestinien?

L. S. - Comme je l'ai dit, le mouvement national est né dans la diaspora, dans la périphérie de la Palestine, y compris à Gaza avant l'occupation de 1967. La base des combattants est principalement assurée par les camps de Gaza, du Liban, de Jordanie et de Syrie. Les cadres viennent aussi de cette diaspora. Arafat était au Koweït, Abou Jihad à Gaza, Mahmoud Abbas au Qatar. Ce mouvement est tellement fort qu'il attire nombre de militants de la gauche du monde arabe, dont beaucoup d'intellectuels. Parmi eux, le grand poète Mahmoud Darwich qui, après avoir quitté la Palestine, se rend à Beyrouth où il devient membre du comité exécutif de l'OLP. Il en est de même d'Edward Said qui le rejoint au comité central de l'OLP. Dans les années 1960-1970, l'élite financière, intellectuelle, technocratique palestinienne qui se trouve dans le Golfe pour aider au fonctionnement de ces nouveaux pays encore démunis de cadres se met au service de l'OLP. L'organisation reçoit donc beaucoup d'argent de la diaspora, ce qui permet notamment de donner des bourses pour étudier à l'étranger ou pour fonder des centres d'études palestiniennes comme l'institut des études palestiniennes fondé par Walid Khalidi. Durant cette période historique, la cause palestinienne est en pleine effervescence.

Il y a aussi les émigrés syro-palestiniens partis avant 1948, les «turcos», essentiellement des chrétiens de Bethléem et des environs qui ont émigré en Amérique latine et en Amérique du Nord. On compte aujourd'hui sur le continent américain plus d'un million de descendants d'émigrés palestiniens. En 1969, l'OLP tient son premier Conseil national, soit l'équivalent d'un parlement en exil, et une vingtaine suivront souvent tenus à Alger. Ces Palestiniens venus d'Amérique (Chili, Brésil, Guatemala et autres pays) avaient souvent perdu leur arabe et l'on parlait alors en anglais ou en espagnol. Cet attachement des turcos à la Palestine est encore très grand, certains d'entre eux se font d'ailleurs construire des villas près de Ramallah où ils viennent l'été pour y passer des vacances, se marier ou faire apprendre l'arabe à leurs enfants. S'ils peuvent faire cela, c'est surtout depuis Oslo, qui permet le retour du secteur privé palestinien de la diaspora. Il investit ainsi dans les projets industriels et crée de l'emploi dans les territoires dirigés par l'Autorité palestinienne.

Avec Oslo et l'installation de l'Autorité palestinienne à Ramallah, le centre du pouvoir est en effet revenu en Palestine, après avoir été surtout exercé dans la diaspora, à Amman, Beyrouth et Tunis. Même si le territoire du futur État ne représentera que $22 \%$ du territoire du mandat, ce retour demeure une grande avancée depuis la nakba de 1948. D'un certain point de vue on territorialise la Palestine. On la ramène chez elle et on ouvre la perspective d'un État. 
Cette mobilisation de la diaspora s'ajoute à une mobilisation internationale. Comment la percevez-vous?

L. S. - La mobilisation internationale est décevante sur le plan gouvernemental mais encourageante sur le plan non gouvernemental, surtout avec la campagne BDS (Boycott-Désinvestissement-Sanctions). Depuis des décennies, les Palestiniens bénéficient de contacts avec des militants venus de l'étranger mais aussi de rencontres avec des intellectuels (universitaires, écrivains, artistes) internationalement connus comme Jean-Luc Godard, Jean Genet ${ }^{2}$ et tant d'autres. Je me souviens d'une réunion à Amman en 1970 où nombre d'intellectuels de premier plan s'étaient pressés autour d'Arafat et des leaders palestiniens pour les protéger contre l'armée jordanienne. Si le combat pour la Palestine a drainé autant de militants internationaux, c'est parce qu'ils percevaient sa justesse. Et c'est encore le cas avec nombre de syndicalistes internationaux, de mouvements de solidarité qui se mobilisent pour défendre les droits des Palestiniens. Je garde le souvenir ému de Stéphane Hessel et sa femme Christiane qui, bien que très âgés, sont venus dénoncer la construction du Mur dans la zone de B'ilin. Je pense aussi à la mobilisation du Parlement des écrivains avec Jacques Derrida, Juan Goytisolo, Bei Dao, Russell Banks, José Saramago, Breyten Breytenbach, Vicente Consolo et Christian Salmon qui, en mars 2002, se sont rendus à Ramallah auprès de Mahmoud Darwich et de Yasser Arafat dans la mouqata assiégée par les Israéliens. Ces mobilisations très fortes ont été et demeurent toujours très importantes pour les Palestiniens qui ne se sentent pas totalement abandonnés. Le Tribunal Russel pour la Palestine en est un très bon exemple.

Mais il faut aussi parler des mouvements pour la paix qui existent en Israël (Alternative Information Center, Breaking the Silence, Les rabbins pour la paix, Les anarchistes contre le mur, etc.). Ainsi, à chaque mois d'octobre lors de la cueillette des olives, des Israéliens forment un cordon de sécurité pour protéger les paysans palestiniens des colons qui tirent sur eux.

Cet engagement d'Israéliens et d'étrangers venus de tous horizons culturels et politiques est une chance pour une société palestinienne dont l'histoire a été marquée par le pluralisme et le cosmopolitisme, depuis la présence des missions étrangères à Jérusalem à la fin du XIX siècle jusqu'à aujourd'hui. Cette dimension pluraliste a une profondeur en Palestine, ne serait-ce que parce qu'elle a été le lieu de beaucoup de prophéties et de miracles!

Quand je regarde cette histoire et sa richesse, je suis très inquiète de la situation actuelle que l'on nous présente comme une guerre de civilisation où il n'est pas possible de vivre la différence. Ce que souhaite faire croire la propagande Daech,

2. Jean Genet, Un captif amoureux, Gallimard, Paris, 1995. 
et Netanyahou aussi, c'est bien cela, autrement dit une guerre judéo-chrétienne contre le monde arabo-musulman. Malheureusement, même dans un pays comme la France qui fut si riche de sa connaissance du monde arabe, les intellectuels qui déconstruisent cette représentation ne sont plus toujours entendus. La diffusion de cette idée d'une impossible coexistence est un grand danger pour les Palestiniens qui risquent de perdre définitivement cette caractéristique si précieuse de la société palestinienne: la pluralité. Or le projet de deux États vivant côte à côte en Israël et Palestine est le meilleur antidote à la montée du racisme, de la peur de l'autre et de son rejet. C'est la base de la coexistence et du vivre ensemble au Moyen-Orient et par-delà dans le monde.

Propos recueillis par Béatrice Giblin et Pierre Blanc. 
